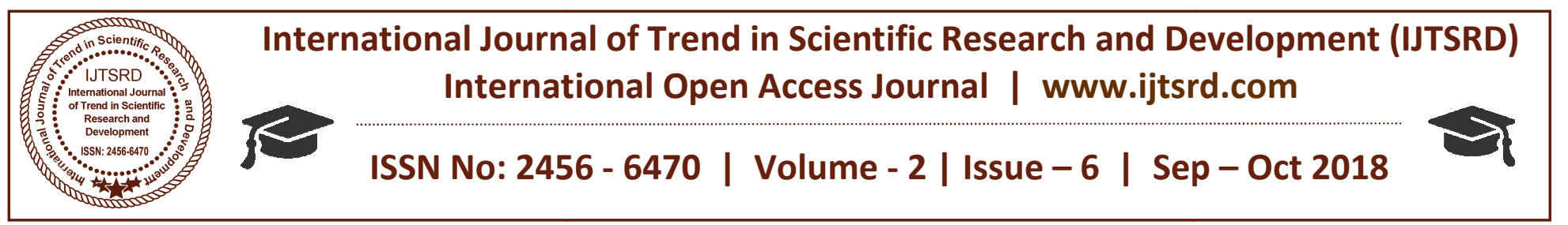

\title{
Sixth Sense Technology
}

\author{
Girubaa. G, Pavithra. M, Deepak. K
}

B.Sc (Information Technology), Sri Krishna Aditya College of Arts and Science,

Kovaipudur, Coimbatore, India

\section{ABSTRACT}

Sixth Sense is a gesture-based wearable computer interface system developed at MIT Media Lab by Steve Mann in 1994 and in 1997 (head worn gestural interface) was developed, and in 1998 (neck worn version), was developed and further Pranav Mistry from MIT MEDIA LAB developed hardware and software for head worn and neck worn versions in the year of 2009. It connects the physical world with digital world around us .It consists of hardware components connected wirelessly to the computing peripherals. It uses enabling surfaces, walls and physical object as interface and it reduces the gap of line between physical and digital world. It help us to take right decision which improve our power of knowledge. Goal is to bring part of the physical world to digital world.

Keyword: Augmented, Gesture, applications.

\section{INTRODUCTION}

Sixth sense technology is a wearable gestural interface which bridges the rupture between real world and digital world physical object and enables us to interact with this using natural hand Sixth sense work is a embellishment like mobile wearable gestures. Sixth Sense is a mini-projector coupled with camera and cell phone. Camera, mirror, projector connected wirelessly to a Bluetooth smart phone that can used comfortably by human beings. Camera recognizes individual images, pictures and gestures and all its details are sent to smart phone for processing. The projector faces downward and project the output image on the mirror so that we can adjust the focus and project on the desired surface. The real power of Sixth Sense lies on its ability to connect the real world with the Internet, and laminate the information on the world and users use natural hand gestures to interact with the information.

\section{HISTORY:}

Sixth sense technology was started late in 1990's by Steve Mann at MIT who actually proposed first wearable computer. First founded a head worn projector and camera in 1994, and then he developed it and proposed neck worn projector and camera during 1998 and further it was developed by Pranav Mistry who is a PhD student in the Fluid Interfaces Group at MIT Media Lab. Steve Mann is regarded as "FATHER OF EMERGENCE SIXTH SENSE" technology. The first architecture of sixth sense was very much bigger and was not working properly to use daily so they came out with a modified neck worn type which was like a pendant. The archetype was built from an ordinary webcam and a battery-powered $3 \mathrm{M}$ projector with has attached mirror and being connected to an internet-enabled mobile phone.

\section{WHY SIXTH SENSE:}

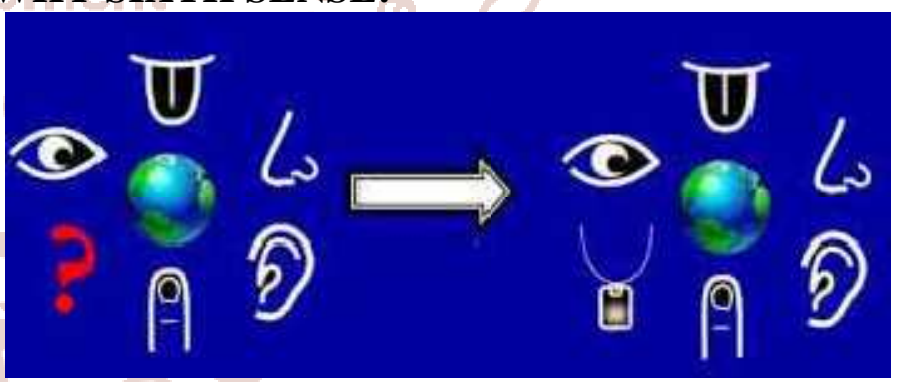

Five natural senses are in need to take information from the world and take decision and action. Information can be collected by using mobile, computer etc. But five senses is just not enough to give the write result, there is no defined relation between physical object and real world. Information is described traditionally on paper or digitably on screen. To come up with this drawbacks sixth sense technology evolved. It gives us freedom to interact with physical world by mere hand gesture. Mostly used in artificial intelligence and this methodology can help develop robots that will interact with humans. 


\section{COMPONENT:}

The hardware components are merged in a pendant like mobile wearable device.

\section{CAMERA:}

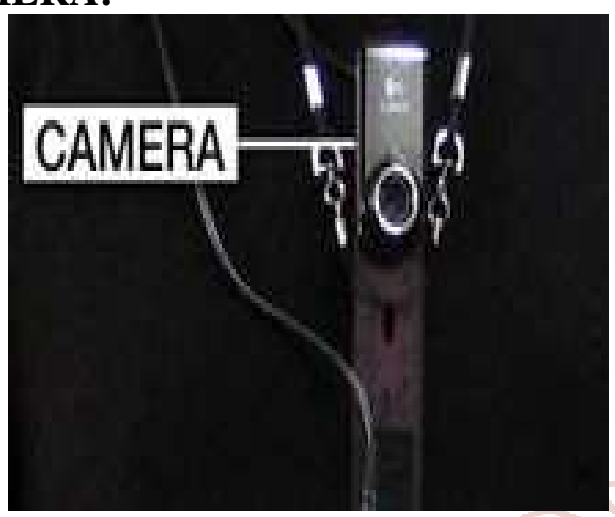

Captures an object for view and tracks the user's hand gestures. It sends the data to smart phone. It acts like a digital eye, connecting you to the world of digitalization.

\section{PROJECTOR:}
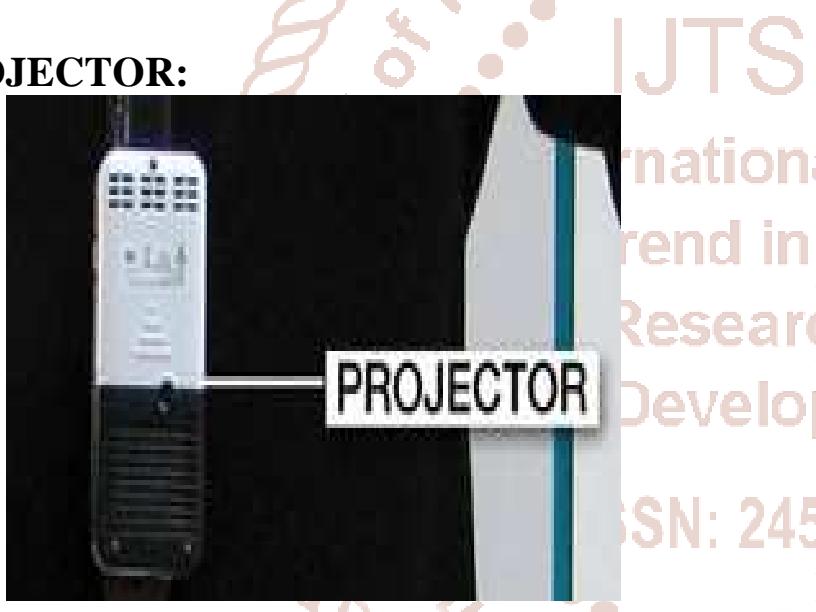

Projects visual information enabling surfaces and physical objects to be used as interfaces. The project contains a battery inside, with 3 hours of battery life. A tiny LED projector displays data sent from the smart phone on any surface such as View-object, wall, or person.

\section{MIRROR:}

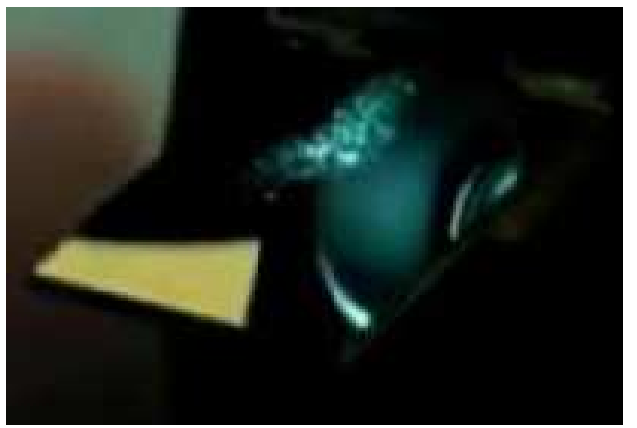

The usage of the mirror is similar as projector swings loosely pointing downwards from the neck.

\section{SMART PHONE:}

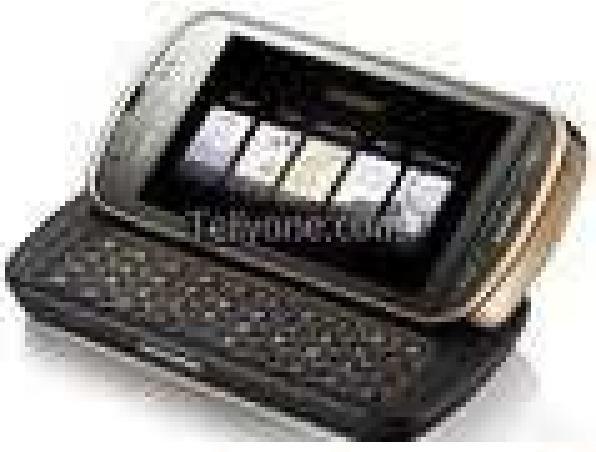

A Web-enabled smart phone in the user's pocket processes the video data. Other software searches the Web and interprets the hand gestures.

\section{COLOR MARKERS:}

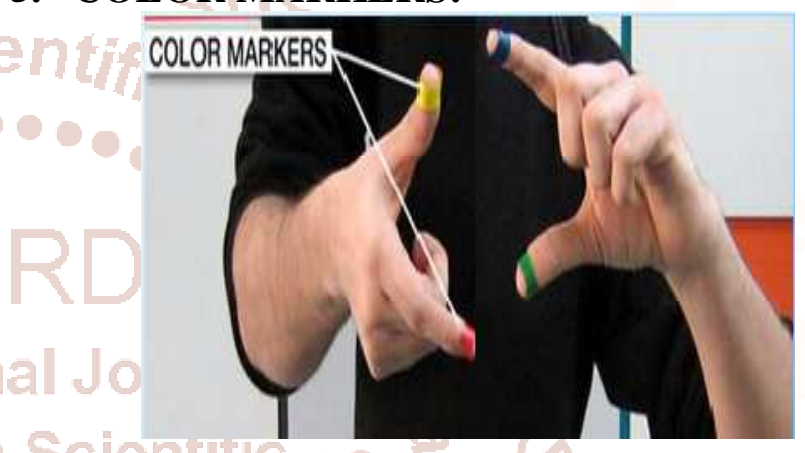

It is at the nook of user's fingers. Marking the user's fingers with red, yellow, green, and blue tape helps the webcam recognize gestures. The movements and arrangements of these makers are interpreted into gestures.

\section{HOW DOES IT WORKS:}

The peripheral that makes Sixth Sense work is a pendant like mobile wearable interface. It has a camera, a mirror and a projector and is connected without wire to a Bluetooth smart phone the camera identifies individuals, images, pictures, gestures one makes with their hands. Information is sent to Smartphone for process. The projector which is facing down projects the output image on to the mirror which reflects image on to the planned surface. Thus, digital information is freed and placed in the physical world.

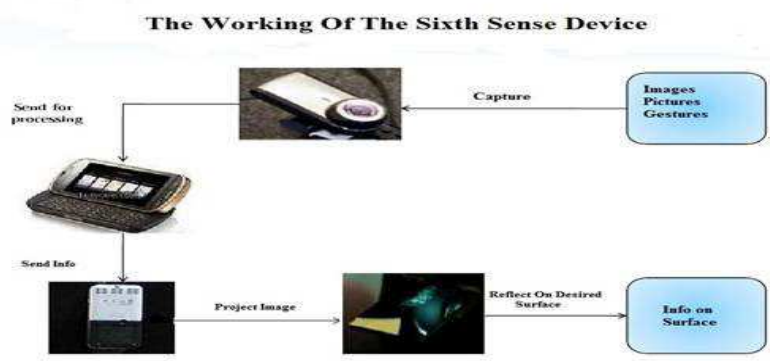




\section{LATEST TECHNOLOGY:}

Currently a laser projector with a laser diode is used which projects inside on any surface. The interesting fact about laser projector is that is never goes out of focus. Since the application been suggested in the interface requires user to wear projector on their body and so laser projector become more popular as there is no need to adjust focus.

\section{APPLICATIONS:}

\section{MAKE A PHONE CALL:}

You can use the Sixth Sense to view a keypad on your hand, and then use that virtual keypad to make a call.

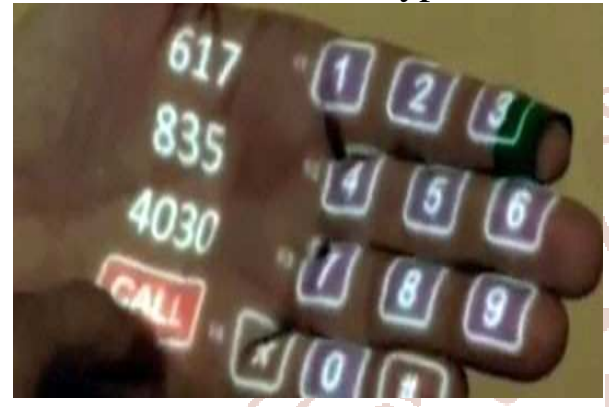

\section{MAP CALL:}

User can call map function which enables the user to navigate a map displayed on a nearby surface using hand gestures. With the map application user can call up the map and use thumbs and index fingers to navigate the map.

\section{CHECK YOUR'S TIME:}

Virtual watch gives you the correct time, on your wrist which has a circle drawn by us.

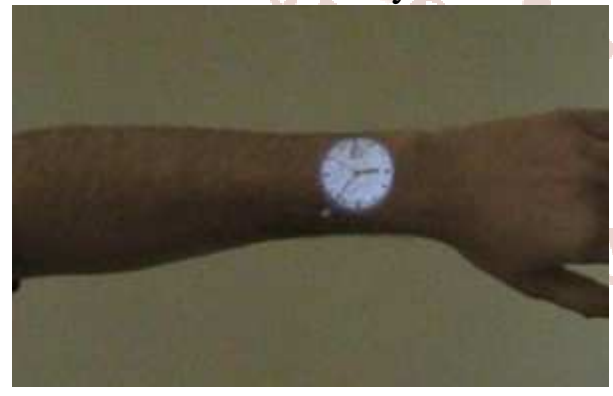

\section{CREATE MULTIMEDIA TECHNOLOGY:}

Sixth Sense can be programmed as videos on the newspaper articles as it makes details so interactive

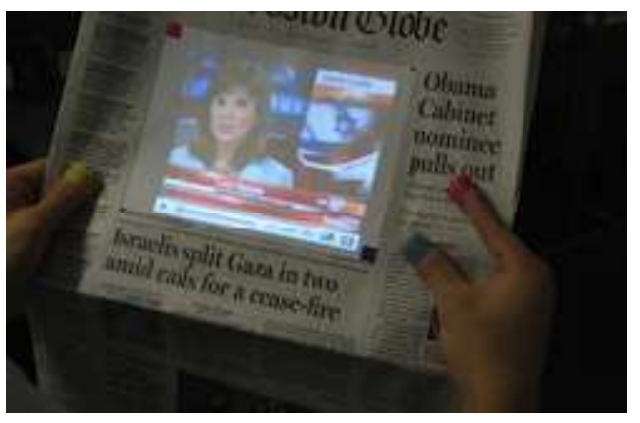

\section{APPLICATION DRAWINGS:}

It lets the user draw on any surface by tracking the fingertip movements of the user's subscript.

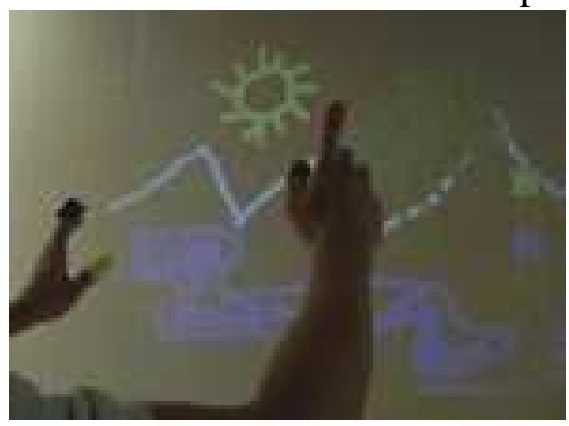

\section{GET FLIGHT UPDATES:}

The system will identifies your boarding pass and let you know whether your flight is on time and if the gate has changed.

\section{TAKE PICTURES:}

If you fashion your index fingers and thumbs into a square the system will snap a photo.

After taking the required number of photos, we can project them onto a surface, and use gestures to sort through the photos, and organize and resize them.

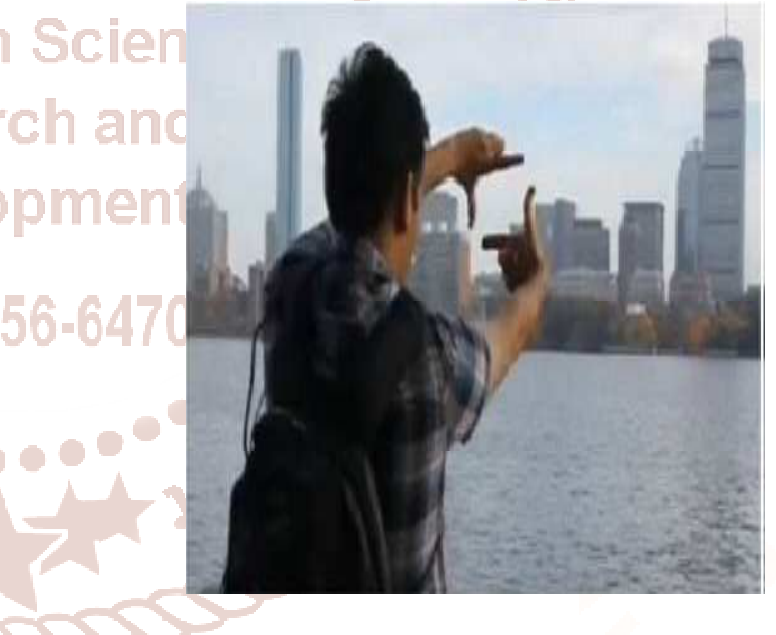

\section{ADVANTAGES:}

1. Portable.

2. Supports multi-touch and multiuser Interaction.

3. Enables Connectedness between world and information.

4. Cost effective only (\$300).

5. Data access directly from machine in real time.

6. It is an open source.

\section{CONCLUSION:}

Sixth Sense recognizes all the objects which surrounds us, displays information automatically and letting the user to access it in any way we need. The Sixth Sense prototype implements several 
applications that demonstrate the usefulness, viability and flexibility of the system allowing us to interact with this information via natural hand gestures the potential of becoming the ultimate "transparent" user interface for accessing information about everything around us enables you the whole world at your fingertips-literally. Transparency between user boundary for accessing information about everything around us and this is classified under wearable computing. Recognize the object and give information about it and give freedom to access.

\section{REFERENCES:}

1. www.youtube.com

2. WWW.wikipedia.org

3. www.google.com

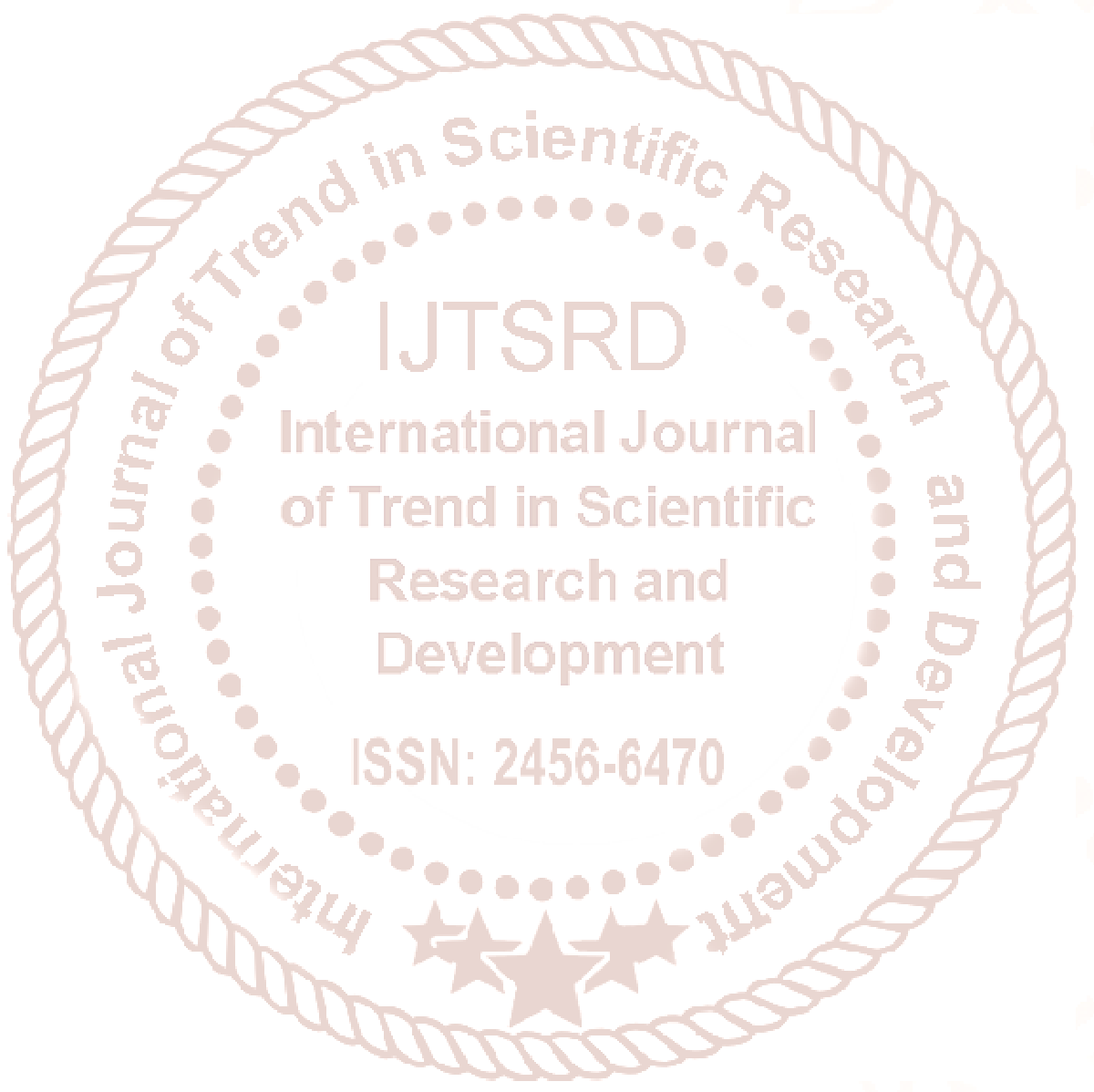

